Beide Bücher zeichnen sich durch eine große Fülle an Informationen aus. Die Arbeit von Weckenbrock, in ihrem Umfang für eine Magisterarbeit sehr ambitioniert, ist inhaltlich stringenter aufgebaut. Eine gesellschaftliche Verständigung über die Möglichkeiten und Grenzen der streitbaren Demokratie und die damit verbundenen Fragen zum Umgang mit verfassungsfeindlichen Parteien bleiben vor dem Hintergrund des anhaltenden Erfolgs der NPD weiter hoch aktuell. Für die inhaltlich umfassende Einarbeitung in die verschiedensten Facetten des Rechtsextremismus in Deutschland ist hingegen die Arbeit von Gerard Braunthal eher zu empfehlen.

Sebastian Rehse

\title{
Gelungene Festschrift für Bonner Gelehrten: Wissenschaftsethos im Praxistest
}

Bracher, Karl Dietrich, Hans-Adolf Jacobsen, Volker Kronenberg und Oliver Spatz (Hrsg.): Politik, Geschichte und Kultur. Wissenschaft in Verantwortung für die res publica. Festschrift für Manfred Funke zum 70. Geburtstag, Bouvier Verlag, Bonn 2009, 429 Seiten, € 49,-.

Würde man heute in einer Art „Praxistest“ Abgeordnete der Landtage, des Deutschen Bundestages oder des Europäischen Parlaments danach fragen, wo sie ihre Ausbildung erhielten, so würde zweifellos die Universität Bonn überraschend oft genannt werden. Das ist keineswegs verwunderlich, denn das Seminar für Politische Wissenschaft der Bonner Universität war über Jahrzehnte von nur an diesem Ort möglichen Besonderheiten geprägt nämlich von den hier sehr engen Beziehungen zwischen Wissenschaft und Politik. Daher blieb das politikwissenschaftliche Seminar in Bonn auch zumeist von den oft sehr realitätsfernen linksdogmatischen, ja fundamentalistischen Ideologisierungen verschont, wie sie anderswo, zumal für die Zeit nach 1968, dominierten. Stets hatten die Studenten und Absolventen des Bonner Seminars die Chance, ihr theoretisches Wissen in der Praxis zu überprüfen und weiterzubilden - vor allem durch ihre Mitarbeit für die Abgeordneten oder im vielfältigen parlamentarischen Betrieb.

Unterstützt wurden sie darin von Gelehrten, die zentrale Forschungsgebiete repräsentierten. Genannt seien hier nur Karl Dietrich Bracher für die zeitgeschichtlichen Aspekte, Hans-Adolf Jacobsen für die internationalen Beziehungen sowie nicht zuletzt Manfred Funke für die politische Ideen- und Theoriegeschichte mit einer ausgeprägten Hinwendung zu aktuellen Problemen wie dem Extremismus oder der Politischen Kultur. Er war es auch, der die Verbindung hielt zur politischen Bildung, indem er - oft gemeinsam mit Bracher und Jacobsen - mehrere, zu einzelnen Fachgebieten grundlegende Sammelbände für die Bonner Bundeszentrale für politische Bildung edierte. Eng verbunden mit dieser publizistischen Arbeit des Seminars war die Politikberatung für Regierung und Parlament, wenngleich diese eher unspektakulär erfolgte.

Durch solche Orientierung an den Erfordernissen politisch-parlamentarischer Praxis sowie an den akademischen Lehr- und Lernbedürfnissen blieb die „Bodenhaftung“ des Bonner Seminars bewahrt. Hinzu kamen die für das politische Bonn charakteristischen Merkmale der Bescheidenheit und des Maßhaltens sowie die Abneigung gegen Extremismen jeglicher Art - glückliche Eigenschaften für die Politikwissenschaft wie für die Politik selbst. 
Aber auch dieses Aufrechterhalten von Werten praktischer Vernunft - zumal für die Kompromisserfordernisse der repräsentativen parlamentarischen Demokratie - musste stets gegen die Versuchungen utopischen, radikalen Denkens verteidigt werden. Nicht von ungefähr warnte vor allem der mit der hier anzuzeigenden Festschrift geehrte Manfred Funke in zahlreichen Schriften und Vorträgen vor den Gefahren einer sich immer stärker ausbreitenden, ja wuchernden Ideologisierung in der Politik, aber leider auch in der Wissenschaft also vor dem, was der Philosoph und Schriftsteller Gerhard Nebel als „Karzinom des Geistes" bezeichnet hat. Diese grundsätzlichen Überzeugungen Funkes seien beispielhaft mit den Titeln zweier seiner Publikationen veranschaulicht: „Kultur der Bescheidenheit. Zum Fundament deutscher Politik in der Allianz“ und „Antifaschismus - Zum Blendcharakter einer politischen Allzweckwaffe“.

Die Konfrontation fundamentalistischer Ideologien mit den Notwendigkeiten einer alltagsnahen politisch-parlamentarischen Praxis sowie überhaupt mit den gesellschaftlichen Realitäten wird im Folgenden anhand zweier Beiträge aus der Festschrift aufgezeigt. Der erste befasst sich mit den auch heute noch äußerst unterschiedlichen Reaktionen auf die „braune“ wie die „rote“ Diktatur in Deutschland; der andere mit einer Interpretation des Abgeordnetenmandats als "öffentliches Amt“.

Als älterer „Zeitzeuge" berichtet der emeritierte Professor Carl-Christoph Schweitzer (zeitweise Abgeordneter des Deutschen Bundestages und später intensiv engagiert in der „Aktion Gemeinsinn“) über „Zwei Vergangenheitsbewältigungen von deutschen Diktaturen im vorigen Jahrhundert“. Er ist einer der letzten Vertreter der „Aufbaugeneration“, der sich von Beginn an für die Neugründung der parlamentarischen Demokratie einsetzte. Schweitzer analysiert ausführlich und pointiert die angebliche „Restauration" während der Adenauerzeit sowie das ebenso angebliche Versagen seiner Generation hinsichtlich der notwendigen Auseinandersetzung mit der NS-Zeit.

Aufgrund seiner lebenslangen politischen und wissenschaftlichen Auseinandersetzung mit beiden deutschen Diktaturen und seines tatkräftigen Eintretens für die Demokratie er war einer der „Gründerväter“ der Bundeszentrale für Politische Bildung - übt Schweitzer entschiedene Kritik daran, wie das Terrorsystem der DDR von einigen westlichen „DDRForschern" beschwiegen oder verharmlost wurde. So vermisst er auch eine adäquate zeitgeschichtliche wie ideologische Einordnung der DDR in die historisch längste Gewaltherrschaft der Weltgeschichte, des Kommunismus, dessen Opferzahl sich nach neuen Schätzungen auf über 100 Millionen beläuft. Die DDR war zweifelsohne ein späterer Teil dieses Schreckenssystems. Insofern ist es ein nicht hinnehmbarer Skandal, wenn diejenigen in Politik, Medien und Wissenschaft, die unablässig den „braunen“ Terror anklagen, den kommunistischen verschweigen.

In dem anderen hier zu erwähnenden Beitrag erörtert der emeritierte Bonner Staatsrechtslehrer Josef Isensee und andere das Selbstverständnis des Abgeordneten in seiner vermittelnden Funktion zwischen Gesellschaft und Staat: Er nehme hier eine Zwitterstellung ein (um nicht zu sagen: eine zwiespältige Rolle). Es ist der bekannte Dualismus, der zu einem für Staat und Gesellschaft akzeptablen Ausgleich gebracht werden muss: Auf der einen Seite das grundgesetzlich definierte, aber mit bestimmten Erwartungen verbundene „öffentliche Amt des Abgeordneten", auf der anderen dessen Freies Mandat in der Konkurrenz politischer Kräfte. Das Amt des Abgeordneten ist also ein „Grenzfall“. Das Grundgesetz, so Isensee, bestimme den Status des Abgeordneten im Amt, „wenn es ihn als Vertreter des ganzen Volkes kennzeichnet, also gerade nicht als Vertreter seiner Partei, seiner Fraktion oder 
seiner Wählerklientel“. Auf der Grundlage des Freien Mandats und des Prinzips der Konkurrenzdemokratie jedoch „entwickelt sich eine parlamentarische Praxis, die dem Amtsprinzip widerstrebt".

Dieser Widerspruch sei unausweichlich. Jeder Abgeordnete müsse hier für sich selber (aber wohl auch hinsichtlich seiner Fraktion) einen Ausgleich finden: Während sich die Mitwirkung an der Gesetzgebung eher am Prinzip des öffentlichen Amtes messen lassen müsse, orientiert sich das nicht minder wichtige Prinzip der Konkurrenzdemokratie an den durchaus andersartigen Normen der parlamentarischen Auseinandersetzung. Dabei sei es, so Isensee, eine ,außerrechtliche Verfassungserwartung“, dass im Alltag der Parlamentsarbeit „Konkurrenzmentalität übergeht in Amtsethos und dass Parteitaktik sich letztlich fügt zum Dienst für das Gemeinwohl“".

Die Festschrift für Manfred Funke zeigt in ihren vielfältigen Aspekten der Analysen von Innen- und Außenpolitik, der Parlamentarischen Demokratie, der Zeit- und Ideologiegeschichte, nicht zuletzt aber auch in der Hinwendung zur politischen Praxis und der politischen Bildung, dass das Bonner Seminar für Politische Wissenschaft die Verantwortung der Wissenschaft gegenüber der Gesellschaft ernst nimmt. Eine solche Qualität auch den entsprechenden Seminaren anderer Universitäten für die Gegenwart und Zukunft zu wünschen, ist eine nahe liegende Schlussfolgerung nach der Lektüre dieses inhaltsreichen Bandes.

Klaus Wippermann 